\title{
Eco-Friendly Extraction of Sinapine From Residues of Mustard Production
}

\section{OPEN ACCESS}

Edited by:

Maria Pilar Bernal,

Center for Edaphology and Applied

Biology of Segura, Spanish National

Research Council (CSIC), Spain

Reviewed by:

Helen Treichel,

Universidade Federal da Fronteira Sul,

Sabine Houot,

Institut National de la Recherche

Agronomique (INRA), France

*Correspondence:

Amandine L. Flourat

amandine.flourat@agroparistech.fr

Florent Allais

florent.allais@agroparistech.fr

Specialty section:

This article was submitted to

Waste Management in

Agroecosystems,

a section of the journal

Frontiers in Sustainable Food Systems

Received: 30 July 2018 Accepted: 12 February 2019

Published: 04 March 2019

Citation:

Flourat AL, Willig G, Teixeira ARS and Allais F (2019) Eco-Friendly Extraction of Sinapine From Residues of Mustard

Production.

Front. Sustain. Food Syst. 3:12. doi: 10.3389/fsufs.2019.00012

\author{
Amandine L. Flourat*, Gaëlle Willig, Andreia R. S. Teixeira and Florent Allais* \\ URD Agro-Biotechnologies Industrielles, CEBB, AgroParisTech, Pomacle, France
}

In this study, our efforts were focused on the optimization of phenolic compounds-mainly sinapine-extraction from residues of industrial mustard production. A preliminary work was conducted to discuss the way data from Folin-Ciocalteu analytical method are reported and exploited in the literature, and to determine whether methanol can be replaced by ethanol as extracting solvent. As this first question had found a positive answer, an optimization of the extraction process on crude and pretreated industrial residue was achieved using a Response Surface Methodology (RSM). Optimal conditions obtained through this Design of Experiments (DoE) allowed the extraction of more than $10 \mathrm{mg} / \mathrm{g}$ of defatted and dried matter (aka DDM) of phenolics from defatted and dried matter, and $13 \mathrm{mg} / \mathrm{g}$ DDM of phenolics from non-pretreated matter (results expressed as equivalent of DDM).

Keywords: mustard residues, sinapine, phenols extraction, response surface methodology, optimization, FolinCiocalteu method

\section{INTRODUCTION}

Valorizing by-products from agriculture is one of the potential ways to achieve a sustainable economy. For instance, in 2016, 2.5 billion tons of cereals and nearly 1 billion ton of oilseeds have been produced thus generating a large amount of by-products (e.g., straw, bran, press cake). According to OCDE/FAO (2015), the agricultural production will grow in the next decade $c a$. 3\% (Western Europe) to reach, in certain area, an increase of 20\% (Middle-East and North Africa) (OCDE/FAO, 2015). Numerous research projects are carried out to convert biomass into fuels (1st or 2nd generations) but also to extract molecules of interest. In the latter case, phenolic compounds are often targeted due to their aromatic structure and their numerous biological activities (e.g., antiradical, antimicrobial, anti-aging, anti-UV, and anti-carcinogenic).

In this study we were more particularly interested in mustard species, such as Brassica juncea, Sinapis alba, Brassica nigra, belonging to the Brassicaceae family that also includes rapeseed and canola. All these plants are known to be rich in phenolic compounds especially in sinapine, the choline ester of sinapic acid. The latter is particularly of interest as it has been used in our research group as platform molecule for the chemo-enzymatic synthesis of an anti-UV agent (Dean et al., 2014; Baker et al., 2016; Luo et al., 2017), a non-endocrine disruptive antiradical additive (Jaufurally et al., 2016) and a bisphenol A substitute for polymers/resins synthesis (Janvier et al., 2017a,b). With regards to these promising sinapic acid-based chemicals, and our region being a major producer of canola and mustard, we dedicated ourselves to the optimization of a sustainable extraction process allowing the recovery of sinapine and sinapic acid from mustard bran locally processed by Charbonneaux-Brabant, a local mustard producer. As sinapic acid costs around $\$ 1,000 / \mathrm{kg}$ (Enovation Chemicals, 2018), this extraction will bring additional value to the residues before its end-use in methanisation or as feed. 
Various extraction methods were reported to extract different parts of the mustard such as seed (Amarowicz et al., 1996; Kozłowska et al., 2016; Thangi et al., 2016), flour (ThiyamHolländer et al., 2014), leaf (Santos et al., 2014; Park et al., 2017), hull and cotyledon (Mayengbam et al., 2014), or meal (Engels et al., 2012; Dubie et al., 2013). The most popular technique uses a mixture of methanol or ethanol in water (Song et al., 2010; Huang et al., 2012; Li et al., 2012; ThiyamHolländer et al., 2014; Park et al., 2017), and often coupled to ultrasound (Thiyam et al., 2004; Terpinc et al., 2012; Dubie et al., 2013; Mayengbam et al., 2014; Szydlowska-Czerniak et al., 2015). Some papers also described extraction methods involving only osmotic water (McCue et al., 2005; Thangi et al., 2016), pressurized solvent (Santos et al., 2014), a mixture of methanol and chloroform (Ishtiaque et al., 2013; Kozłowska et al., 2016), or a mixture of acetone, or methanol and $0.1 \%$ formic acid (Engels et al., 2012). Although the total phenolic content for these different extractions was usually measured using Folin-Ciocalteu method, the standard used to express the total of phenolic content was not always the same. Indeed, while gallic acid and sinapic acid were the two main standards, catechol (Surendra et al., 2012), or chlorogenic acid (Park et al., 2017) were also used. Therefore, because of the diversity of starting materials, extraction processes and analytic method and standards, comparing the phenolic contents from mustard reported in the literature did not seem easy or relevant.

In this study, our attention was focused on the optimization of the phenolics extraction from industrial mustard residue while determining the most relevant phenolic analytical standard among those usually used in the literature.

\section{MATERIALS AND METHODS}

\section{Materials}

Industrial mustard residue was kindly furnished by Charbonneaux-Brabant (Reims, France); two lots, lot 1 and lot 2, were furnished in May 2015 and December 2015, respectively, and analyzed by URD ABI an In Vivo. Ethanol, methanol, hexane and sodium carbonate were purchased from VWR. Folin-Ciocalteu reagent was purchased from Merck. Concentrations were conducted on Rotavap R-300 Buchi under vacuum generated by a PC 3001 Vario ${ }^{\text {pro }}$ from Vacuubrand. Ultrasound-activated extractions were conducted in VWR USC600T. Folin-Ciocalteu reactions were conducted in an oven from Binder. Centrifugations were performed using a Heraeus Fresco 21 from Thermoscientific. UVvisible spectra were recorded on Agilent Cary 60. Design of experiment was conducted in an incubator MaxQ4000 from Thermoscientific. Formic acid and Acetonitrile LC-MS grade was purchased from ThermoFisher Scientific. MilliQ water was produced by Milli-Q Direct 8 from Merck Millipore (Burlington, USA).

Abbreviations: DDM, Defatted and Dried Matter; GAE, Gallic Acid Equivalent; SAE, Sinapic Acid Equivalent; ESE, Ethyl Sinapate Equivalent.

\section{Methods}

\section{Defatting and Drying}

Reactions were conducted in triplicate; $50.0 \mathrm{~g}$ of mustard residue were placed in a soxhlet apparatus. Extraction was performed with $200 \mathrm{~mL}$ of boiling hexane overnight. At the end of extraction, the solid residue was rinsed with $100 \mathrm{~mL}$ of hexane. Organic layers were combined and concentrated under vacuum to yield an yellow-orange oil (Lot 1: 2.58, 2.79, and $2.83 \mathrm{~g}$; Lot 2: $2.58,2.66$, and $2.71 \mathrm{~g}$ ). Solid was placed in an oven at $104^{\circ} \mathrm{C}$ for $72 \mathrm{~h}$ and weighted (Lot 1: 21.2, 21.5, and $20.8 \mathrm{~g}$; Lot 2: 21.8, 21.9, and $22.3 \mathrm{~g}$ ) (Table 1).

\section{Folin-Ciocalteu Assays}

General procedure: $625 \mu \mathrm{L}$ of water, $125 \mu \mathrm{L}$ of Folin-Ciocalteu reagent and $25 \mu \mathrm{L}$ of sample were introduced in a $2 \mathrm{~mL}$ eppendorf and vortexed. One milliliter of a 19\% solution of sodium carbonate $\left(\mathrm{Na}_{2} \mathrm{CO}_{3}\right)$ was added, the mixture was vortexed and incubated for $30 \mathrm{~min}$ at $40^{\circ} \mathrm{C}$ before reading of the absorbance at $765 \mathrm{~nm}$.

Procedure for the establishment of the calibration curves for standards: $15 \mathrm{mg}$ of standard (gallic acid, sinapic acid, ethyl sinapate, ferulic acid, or syringaldehyde) were dissolved in $25 \mathrm{~mL}$ of ethanol and the resulting solution was successively diluted to provide height concentrations $(600,500,400,300,200,100$, 50 , and $25 \mathrm{mg} \cdot \mathrm{mL}^{-1}$ ). These solutions were then submitted to the Folin-Ciocalteu procedure described above to determine the corresponding absorbance and establish the calibration curve.

\section{Solvent Extraction}

Three conditions of extraction were tested, both on dried and defatted mustard residue and on crude residue (Table 2).

For each experiment, $2.3 \mathrm{~g}$ of crude (or $1.0 \mathrm{~g}$ of defatted and dried matter) was introduced in a $50 \mathrm{~mL}$ flask and $9 \mathrm{~mL}$ of solvent $(\mathrm{MeOH}$ or $\mathrm{EtOH}, 70 \%$ in water) was added either at room temperature, at $70^{\circ} \mathrm{C}$, or in the ultrasonic bath. After $30 \mathrm{~min}$, the supernatant was removed and fresh solvent was added. The solid residue was extracted 3 times. At the end, all layers were combined and centrifuged at 5,000 g during $10 \mathrm{~min}$ at $4{ }^{\circ} \mathrm{C}$. Phenolic content of supernatant was evaluated using the Folin-Ciocalteu method as described in section Folin-Ciocalteu

TABLE 1 | Composition of mustard residues.

\begin{tabular}{llcccc}
\hline Residue & $\begin{array}{l}\text { Analysis } \\
\text { performed by }\end{array}$ & $\begin{array}{c}\text { Water } \\
\text { content }\end{array}$ & Fat & Protein & Minerals \\
\hline Lot 1 & URD ABI & $53.73 \%$ & $11.9 \%$ & $\#$ & $\#$ \\
Lot 2 & URD ABI & $52.17 \%$ & $11.1 \%$ & $\#$ & $\#$ \\
$\begin{array}{l}\text { Year 1999 } \\
\text { sample* }\end{array}$ & Charbonneaux & $53.92 \%$ & $17.9 \%$ & $8.16 \%$ & $5.86 \%$ \\
$\begin{array}{l}\text { Year 2005 } \\
\text { sample* }\end{array}$ & Charbonneaux & $51.73 \%$ & $16.2 \%$ & $8.06 \%$ & $6.31 \%$ \\
Lot 1 & In vivo & & & & \\
\hline
\end{tabular}

${ }^{\star}$ Representative samples for the 1999 and 2005 productions. \# no data available. 
TABLE 2 | Results of the non-optimized extractions performed on mustard residues.

\begin{tabular}{|c|c|c|c|c|c|c|c|}
\hline Solvent & Extraction process & Mustard form & $\begin{array}{c}\text { Mass after extraction } \\
\text { and drying (g) }\end{array}$ & Abs at $765 \mathrm{~nm}$ & [C] ESE* (mg/L) & [C] ESE* $(\mathrm{mg} / \mathrm{g})$ & Entry \\
\hline \multirow[t]{6}{*}{ MeOH/Water (70/30) } & Room temperature & Dried and defatted & 0.684 & 0.3036 & 253.0 & 6.83 & 1 \\
\hline & & Crude & 0.821 & 0.1674 & 139.5 & 3.77 & 2 \\
\hline & $75^{\circ} \mathrm{C}$ & Dried and defatted & 0.638 & 0.4555 & 379.6 & 10.25 & 3 \\
\hline & & Crude & 0.867 & 0.2092 & 174.3 & 4.71 & 4 \\
\hline & Ultrasonic bath & Dried and defatted & 0.736 & 0.3222 & 268.5 & 7.25 & 5 \\
\hline & & Crude & 0.753 & 0.2741 & 228.4 & 6.17 & 6 \\
\hline \multirow[t]{6}{*}{ Ethanol/water (70/30) } & Room temperature & Dried and defatted & 0.700 & 0.2756 & 229.7 & 6.20 & 7 \\
\hline & & Crude & 0.865 & 0.1374 & 114.5 & 3.09 & 8 \\
\hline & $75^{\circ} \mathrm{C}$ & Dried and defatted & 0.619 & 0.4525 & 377.1 & 10.18 & 9 \\
\hline & & Crude & 0.853 & 0.2791 & 232.6 & 6.28 & 10 \\
\hline & Ultrasonic bath & Dried and defatted & 0.729 & 0.4079 & 339.9 & 9.18 & 11 \\
\hline & & Crude & 0.774 & 0.3237 & 269.8 & 7.28 & 12 \\
\hline
\end{tabular}

${ }^{*}[C]$ ESE: concentration in phenolic compounds in Ethyl Sinapate Equivalent.

Assays. The solid matter was dried in an oven at $104^{\circ} \mathrm{C}$ overnight and weighted.

\section{Experimental Design and Statistical Analysis}

The effect of the five independent variables $X_{1}$, Temperature; $X_{2}$, Time; $X_{3}$, \% of EtOH in water; $X_{4}$, Solvent/matter ratio, that are scaled and centered; and $X_{5}$, Pretreatment of the raw material, not $(\mathrm{N})$ or defatted and dried (DD)-Table 3 -on the response $(Y$, phenolic contents) was modeled using a polynomial response as described previously (Teixeira et al., 2016).

Response Surface Methodology (RSM), based on 5 factors and 4 central points D-Optimal design, was used to determine the parameters affecting the extraction of mustard residue and to find the optimal set of conditions (SM Table 1). Modde v.12.0 software (Umetrics AB, Sweden) was used to generate the DOptimal design and analyze experimental data by response surface methodology (RSM).

Additional runs (intermediary points) were performed to validate the model and later added to the experimental data for model refining (SM Table 2). D-Optimal design has the advantage to be able to carry qualitative factors, take correlations in count and can be completed by additional experiments if needed.

\section{UHPLC-DAD-ESI-MS Analysis}

UHPLC-MS analysis was performed on a 1,290 infinity II coupled with a 6545Q-Tof-MS from Agilent Technologies (Santa Clara, USA) fitted with dual ASJ-ESI source. UV-visible spectra were recorded at $320,285,254$, and $210 \mathrm{~nm}$ ( $4.0 \mathrm{~nm}$ bandwidth, $2.5 \mathrm{~Hz})$. Mustard extract was separated on Zorbax Eclipse plus C18 (Agilent, Technologies, Santa Clara, USA) $1.8 \mu \mathrm{m}$, $2.1 * 50 \mathrm{~mm}$ at $48^{\circ} \mathrm{C}$. Five microliter of sample was injected and eluted with $0.1 \%(\mathrm{v} / \mathrm{v})$ formic acid in milliQ water (eluent A) and $0.1 \%(\mathrm{v} / \mathrm{v})$ formic acid in acetonitrile LC-MS grade (eluent B) at a flow rate of $0.45 \mathrm{~mL} / \mathrm{min}$. The elution gradient was $5-10 \% \mathrm{~B}$ (0.99 min), 15\% B (3.19 min), 30\% B (7.44 min), 0\% B (8.51 min), and $5 \% \mathrm{~B}(10.00 \mathrm{~min})$. Positive ion mass spectra were recorded in
TABLE 3 | Independent variables and levels used for D-Optimal design.

\begin{tabular}{lccc}
\hline & \multicolumn{3}{c}{ Level } \\
\cline { 2 - 4 } & $\mathbf{- 1}$ & $\mathbf{0}$ & $\mathbf{1}$ \\
\hline \multicolumn{2}{l}{ QUANTITATIVE VARIABLES } & & \\
Temperature & 30 & 42.5 & 55 \\
Time & 0.5 & 3.25 & 6 \\
$\%$ of EtOH & 30 & 65 & 100 \\
Solvent/matter & 10 & 30 & 50 \\
QUALITATIVE VARIABLE & None & & Dried and defatted \\
Pretreatment & & &
\end{tabular}

the range of $m / z 50-1,000$ (scan rate: 2 spectra/sec) with a nozzle voltage at $2,000 \mathrm{~V}$. Nitrogen was used at gas source at $8 \mathrm{~L} / \mathrm{min}$ and $325^{\circ} \mathrm{C}$.

\section{RESULTS AND DISCUSSION}

\section{Composition of Mustard Residue}

Charbonneaux-Brabant uses mostly seeds of Brassica juncea (ca. 90\%) but also Sinapis alba from Canadian and French suppliers. After mustard making, a residual fraction was generated containing mainly the bran but also residual crushed seed depending on the grinning applied for the different mustards produced. In this study a representative mix of the different residues, furnished by Charbonneaux-Brabant, was employed to determine the average content of phenolics from this supplier.

Characterization of this raw material (Table 1) has shown that water content were $53.73 \%$ and $52.17 \%$ for lot 1 and lot 2, respectively, far from the result reported by Dubie et al. (2013) from their "meal remaining after cold pressing of $B$. juncea" with around $5 \%$ of water content. Soxhlet extraction using hexane revealed a residual fat proportion of 5.49 and $5.3 \%$, for lot 1 and lot 2, respectively, corresponding to 11.9 and $11.1 \%$ with regards to dry matter. To accurately report our raw matter to what was often described (i.e., dried and 
defatted mustard), one can thus consider that $2.3 \mathrm{~g}$ of crude product are equivalent to $1 \mathrm{~g}$ of dried and defatted material (DDM). These results were confirmed by data obtained from an external analytical lab (in vivo) that also measured the protein content at $8.8 \%$. It is noteworthy to mention that analyses performed by Charbonneaux-Brabant on their residue in 1999 and 2005 showed good adequacy with our results and those from In vivo (Table $\mathbf{1}$ ).

\section{Folin-Ciocalteu Methodology}

In the Folin-Ciocalteu method, the total phenolic content is always reported as an equivalent against a standard compound. Gallic acid is widely used as representative "equivalent," aka gallic acid equivalent or GAE, to express this phenolic content.(McCue et al., 2005; Song et al., 2010; Huang et al., 2012; Terpinc et al., 2012; Santos et al., 2014; Kozłowska et al., 2016; Thangi et al., 2016). However, in our case, its structure is very different compared to that of sinapine (i.e., three phenol moieties and a benzylic acid for gallic acid, one phenol moiety and an ester for sinapine). In the literature, sinapic acid-that is closer to sinapine in terms of structure-was also employed as standard (i.e., sinapic acid equivalent or SAE) (Amarowicz et al., 1996; Dubie et al., 2013; Mayengbam et al., 2014; Szydlowska-Czerniak et al., 2015). Unfortunately, the use of sinapic acid with the Folin-Ciocalteu method proved troublesome as it did not provide good regression coefficient for the calibration curve. To get more accurate values, five phenolic compounds were thus tested: gallic acid, sinapic acid, ethyl sinapate, syringaldehyde, and ferulic acid (Figure 1). The calibration curves obtained for these compounds are presented in Figure 2. These molecules were chosen to evaluate the impact of the chemical structure of the standards on their reactivity toward Folin-Ciocalteu reagent. Ferulic acid, that possesses only one methoxy group, was used to determine the importance of the substitution of the aromatic ring (sinapic acid derivatives possess two methoxy groups). Syringaldehyde was selected to evaluate the influence of the substituent at the para-position (i.e., aldehyde vs. acid vs. $\alpha, \beta$-unsaturated acid/ester) while ethyl sinapate served as model for esters of sinapic acid. Sinapic acid, and gallic acid being commonly used as standards, they served as benchmarks.

Data showed that, if concentration was expressed in $\mathrm{mmol} / \mathrm{L}$ (Figure 2A), which was rarely reported, gallic acid surprisingly reacted with the Folin-Ciocalteu reagent in a similar fashion than sinapic acid or ethyl sinapate, whereas syringaldehyde or ferulic acid showed significantly lower absorbance at the same concentration. Indeed absorbance coefficients were between 0.30 and 0.33 for sinapic acid, ethyl sinapate and gallic acid whereas they were 0.15 and 0.22 for syringaldehyde and ferulic acid, respectively. These results showed clearly that the substitution of the aromatic ring was an important factor as ferulic acid and sinapic acid derivatives presented different reactivities. In addition, carboxylic acid or ester moieties conjugated with the aromatic ring (i.e., sinapic acid, gallic acid, and ethyl sinapate) showed a different interaction with Folin-Ciocalteu reagent than the aldehyde moiety of syringaldehyde. Finally, as ethyl sinapate, an ester of sinapic acid, reacted in the same way as sinapic acid itself, one can assume that sinapine, another ester of sinapic acid, will also show the same trend.

When the concentration is expressed in $\mathrm{mg} / \mathrm{L}$ (Figure 2B), which is how values are reported most of the time in the literature, one can see that the curve for gallic acid was no longer similar to that of sinapic acid or ethyl sinapate. This is due to the difference of molecular mass of the different compounds, 252.27 $\mathrm{g} / \mathrm{mol}, 224.21 \mathrm{~g} / \mathrm{mol}$, and $170.13 \mathrm{~g} / \mathrm{mmol}$ for ethyl sinapate, sinapic acid and gallic acid, respectively. For example, for 0.5 in absorbance, the total phenolic content will be $258.8 \mathrm{mg} / \mathrm{L} \mathrm{GAE}$ vs. $416.7 \mathrm{mg} / \mathrm{L}$ ESE (Ethyl Sinapate Equivalent), demonstrating that the choice of standard molecule strongly impacts the order of magnitude of the phenolic content. Therefore, to provide a more relevant and accurate phenolic content, it is crucial to use a standard that not only reacts similarly to the considered compound in terms of absorbance but also has a molecular weight close to that of this compound.

Usually, sinapine $\left(\mathrm{M}_{\mathrm{w}}=310.37 \mathrm{~g} / \mathrm{mol}\right)$ is described as the major product of phenolic extracts from mustard. This results has been also observed in our residues by performing HPLC-MS analysis at $320 \mathrm{~nm}$ that clearly shows that the highest peakat $3.89 \mathrm{~min}$-corresponds to sinapine with an isotopic massif centered at $310.2178 \mathrm{~m} / z$ for an exact mass reported at 310.1654 (Da) (Figure 3). By using the extinction coefficient of sinapine at $\mathrm{pH} 5.46$ and $\lambda_{\max }$ of $326 \mathrm{~nm}$ (i.e., $21.350 \mathrm{~mol}^{-1} . \mathrm{cm}^{-1} \mathrm{~L}$ ) reported by Smyk (2015), the average sinapine content of our extract can be determined. For a sample with a concentration of $1.02 \mathrm{mmol} . \mathrm{L}^{-1}$ of phenolic compounds (determined by FolinCiocalteu), sinapine content was evaluated at $0.52 \mathrm{mmol.L} \mathrm{L}^{-1}$, which represents $51 \% \mathrm{~mol}$ of the total phenolic content. Converting these values in mass ratio leads to a total phenolic content of $9.00 \mathrm{mg}$ ESE/g DDM with sinapine representing 5.65 $\mathrm{mg} / \mathrm{g} \mathrm{DDM}(63 \%)$. It is noteworthy to mention that this ratio is similar to that reported by Thiyam-Holländer et al. (2014) on flour of mustard but lower than those reported for cotyledon, hull, and seed by Mayengbam et al. (2014) (70-99\%).

Folin Ciocalteu is a convenient method to determine total phenolic content. However, the standard to interpret/report the results have to be carefully chosen. Indeed, it has to react in a similar way with Folin Ciocalteu reagent than the representative compound(s) of the extract. In addition, to express the results in mass concentration $(\mathrm{mg} / \mathrm{L})$, the molecular weight of the standard has to be in adequacy with that of the targeted molecule(s). Taking these considerations into account, ethyl sinapate was chosen as standard for our study.

\section{Solvent Extractions of Mustard Residues Prior Optimization}

The second step of this work consisted in conducting extraction, both on dried and defatted mustard residue and on crude residue to determine whether methanol could be advantageously replaced by ethanol and to investigate the efficiency of the extraction process (i.e., ultrasound and temperature). It is noteworthy to mention that microwave-assisted extraction was not considered as such treatment can potentially promote the decarboxylation of sinapic acid into canolol (Yang et al., 2014). 

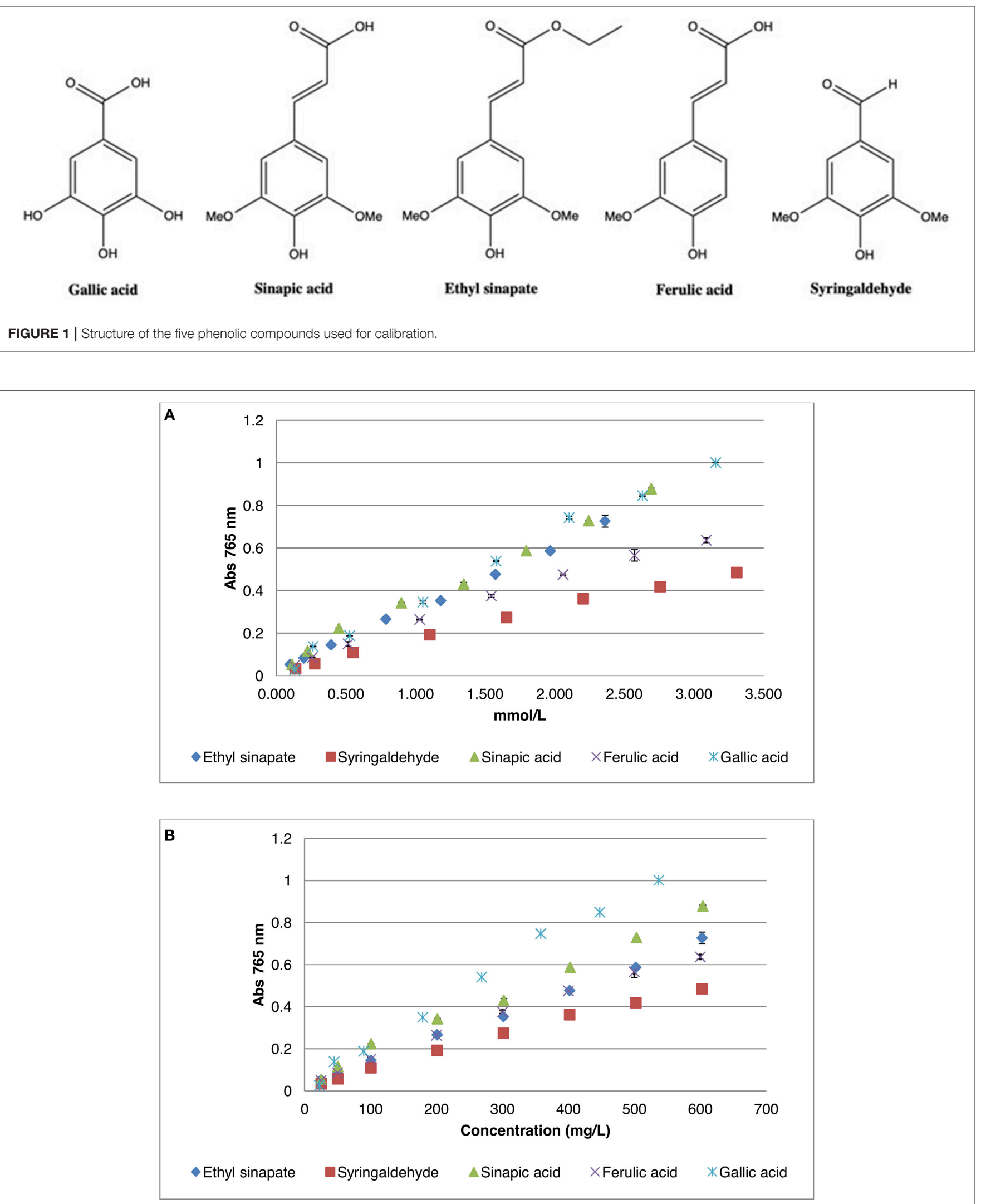

FIGURE 2 | Calibration curves of different phenolic compounds by Folin-Ciocalteu method. (A) Absorbance as a function of the concentration in mmol/L; (B) Absorbance as a function of the concentration in $\mathrm{mg} / \mathrm{L}$. 


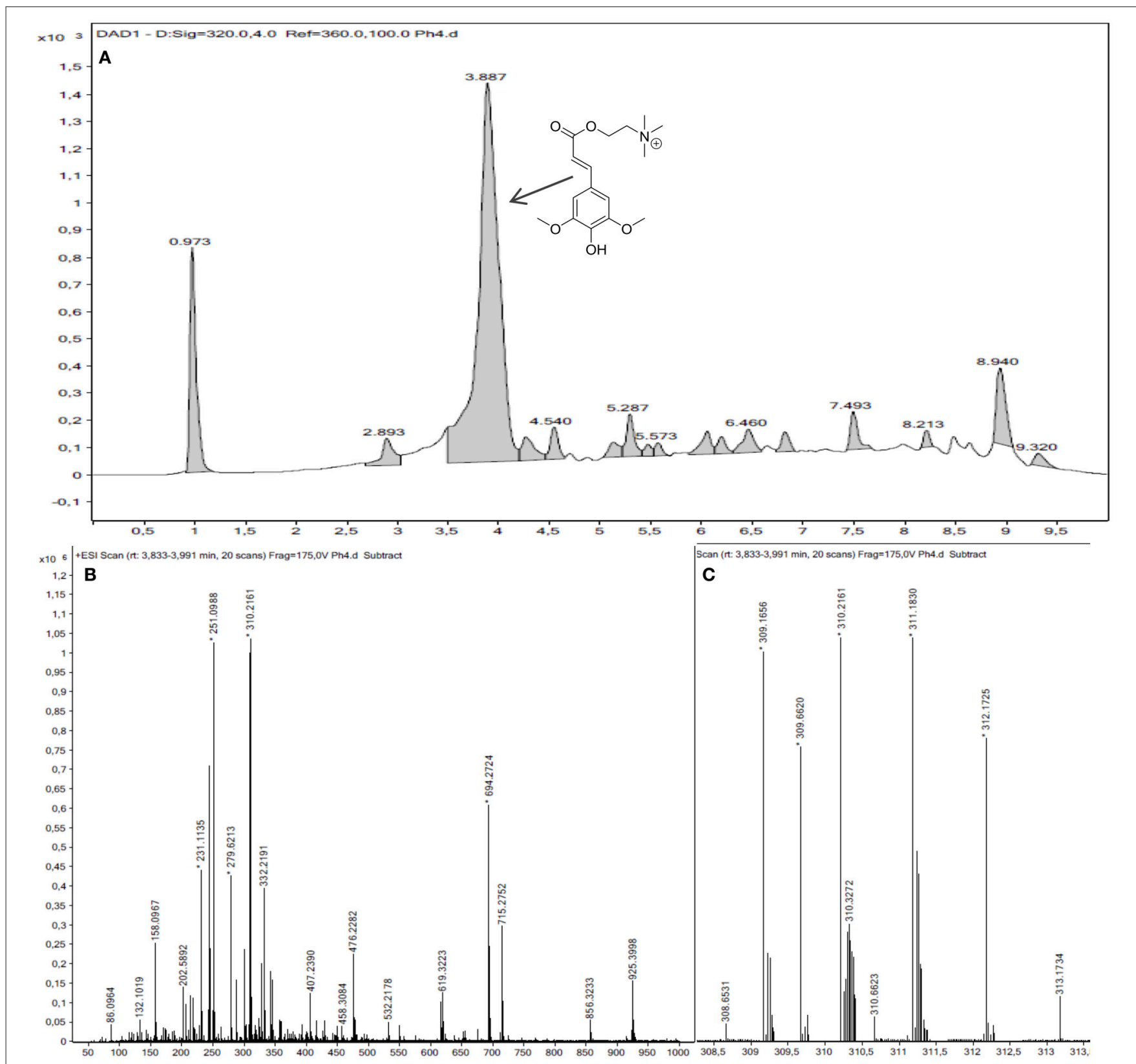

FIGURE 3 | (A) Chromatogram at $320 \mathrm{~nm}$ of mustard bran extract; (B) lonic spectrum of the major compound (Sinapine, $\mathrm{t}_{\mathrm{r}}=3.89 \mathrm{~min}$ ); (C) Zoom on isotopic massif.

The efficiency of the extraction was evaluated by determining the amount of phenolics compounds released using Folin-Ciocalteu method (Table 2).

For both materials (i.e., dried and defatted or crude), one can observe that methanol can be advantageously replaced by ethanol, except when the extraction is conducted at room temperature (Entry $1-2$ vs. 7-8). Indeed, at $75^{\circ} \mathrm{C}$ and under ultrasound, recovered masses after extraction with ethanol were quite similar, and most of all, Folin-Ciocalteu analyses revealed that phenolic contents were higher than that obtained with methanol (Entries 3-6 vs. 9-12). For instance, for crude residue extracted at $75^{\circ} \mathrm{C}$, replacing methanol by ethanol resulted in an increase of $\mathrm{ca}$. $33 \%$ in phenolic content (from 4.71 to $6.28 \mathrm{mg} / \mathrm{g}$ ).

With regards to the extraction mode on both treated or untreated residues, even if recovered mass was quite the same with or without heating thermally the reaction, the phenolic concentration was highly impacted as about twice more phenolics were extracted when the reaction was warmed at $75^{\circ} \mathrm{C}$ compared to room temperature (Entries 1-2, 3-4, 7-8, and 9-10). More than $10 \mathrm{mg} / \mathrm{g}$ can be extracted at $75^{\circ} \mathrm{C}$ from dried and defatted matter. It is worth mentioning that ultrasound did not bring 
any improvement regarding thermal heating on defatted and dried material (Entries 3 and 9 vs. 5 and 11), whereas, for crude residues, results were increased by 24 and 13\% with methanol and ethanol, respectively (Entries 4 and 10 vs. 6 and 12). One possible explanation is that ultrasound provided a better solubilization of the fat leading to a better release of the phenols. This hypothesis is consistent with the fact that recovered masses after extraction and drying were the same for crude matter or defatted and dried one in the case of ultrasound treatment (Entries 5-6 and 11-12), while the fat seems to remain in the solid residue for the thermal process (Entries 3-4 and 9-10).

In summary, these preliminary results demonstrated that (1) ethanol can be used instead of methanol without negatively impacting the phenolic content, and (2) defatted and dried matter releases more phenolic compounds than crude one.

Our objective being to propose an ecological and economical extraction process, the following work aimed at achieving high extracted phenolic content in only one extraction step with ethanol/water to limit both solvent handling and process cost. Moreover, in order to go deeper into this investigation and to determine whether the defatting step was required or not, a Design of Experiments (DoE) was performed.

\section{Optimization of the Extraction by Design of Experiments}

The aim of this DoE was to predict the quantity of phenolics released with regards to five parameters-temperature $(X 1)$, time $(X 2)$, percentage of ethanol in extracting solution $(X 3)$, ratio between solvent and matter $(X 4)$, and pretreatment or not of the mustard residues (X5) (Table 3 ).

After performing the 31 extractions, one outlier (SM Table 1, entry 18) was found. For the regression analysis, a normally distributed response was suitable that is why logarithm transformation was applied to our data. Excellent reproducibility in the central point was reached, 0.994; and $\mathrm{R}^{2}$, fit between experimental data and the model, and $\mathrm{Q}^{2}$, estimation of the future prediction precision, were good as well, 0.926 and 0.714 respectively, but internal validity was negative, -0.14 , this value correspond to the lack of fit of the model (internal error are larger than error from repeated point). To achieve a model with a significance higher than $95 \%, p$-value of regression need to be inferior at 0.05 whereas $p$-value of lack of fit need to be $<0.05$. In our case, the significance of the lack of fit was not reach $(p=0.011)$.

The outlier (18) as well as other "noising" points-(difference between the experimental value and the one predicted by the model)-(Entries 1, 11, 15, 21, 24, 32, 34, and 35), were duplicated (SM Table 2, entry 36-50). With these new values the internal model validity was reached. As a new lot of raw material was used to perform entries 37-50 for the refining of the model, three new central points were tested with this new lot and provided good results compare to reproducibility (SM Table 2, entries 51-53) that were included in the model. It is noteworthy to mention that this new lot was a mix of residues similar to that of lot 1 . Its water and fat content was evaluated as previously described in section Defatting and Drying, representing $56 \%$ of the crude matter. The amount of raw material used in the experiments was adapted in consequence.

All the data except the outlier (SM Table 1, entry 18) were included in the model. With these new data, all the indicators now reached good values, $R^{2}=0.857, Q^{2}=0.742$, Model validity $=0.733$ and reproducibility $=0.833$. Moreover, $F$-tests performed in ANOVA assessing the significance of the regression model $(p<0.05)$ and the lack of fit $(p>0.05)$ showed statistical

\section{Coefficients (scaled and centered)}

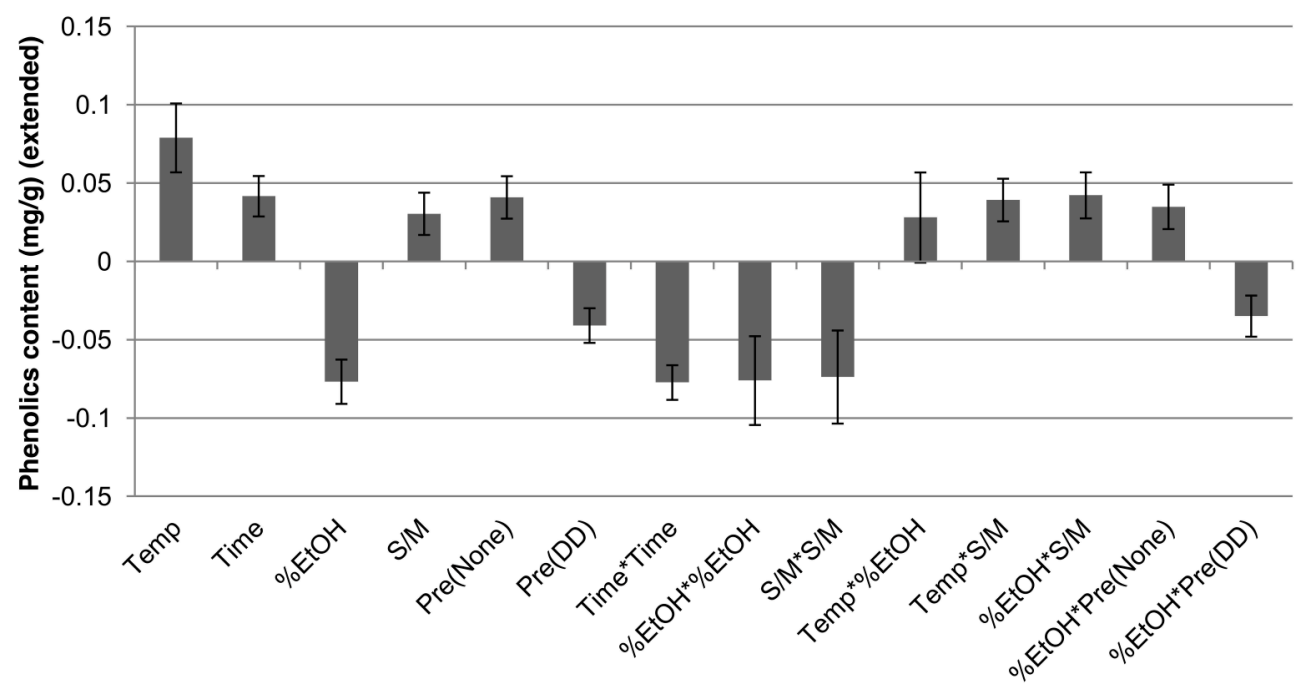

FIGURE 4 | Regression coefficients of phenolic content model. Model coefficients are scaled and centered. 
significance of both models and a similar magnitude of replicate errors (no lack of fit).

The condition number was 6.06 , so in the range $(<8)$ of what was expected to assess the sphericity of the design in optimization. As previously mentioned a logarithmic transformation of the data had been performed to achieve a normal distribution leading to equations of the model below, the first one for the raw material (Equation 1), the second for the defatted and dried material (Equation 2) with unscaled $\mathrm{X}_{i}$ in order to enable the direct application of equations.

$$
\begin{aligned}
& \log (Y)=0.738685-0.0025656 \mathrm{X} 1+0.0815867 \mathrm{X} 2 \\
& +0.00134211 \mathrm{X} 3+0.00199775 \mathrm{X} 4-0.0238607 \\
& \quad-0.0102209 \mathrm{X} 2^{2}-0.0000620895 \mathrm{X} 3^{2}+0.000184466 \mathrm{X} 4^{2} \\
& +0.0000641509 \mathrm{X} 1 \mathrm{X} 3+0.000156992 \mathrm{X} 1 \mathrm{X} 4 \\
& +0.0000603143 \mathrm{X} 3 \mathrm{X} 4+0.000996512 \mathrm{X} 3
\end{aligned}
$$

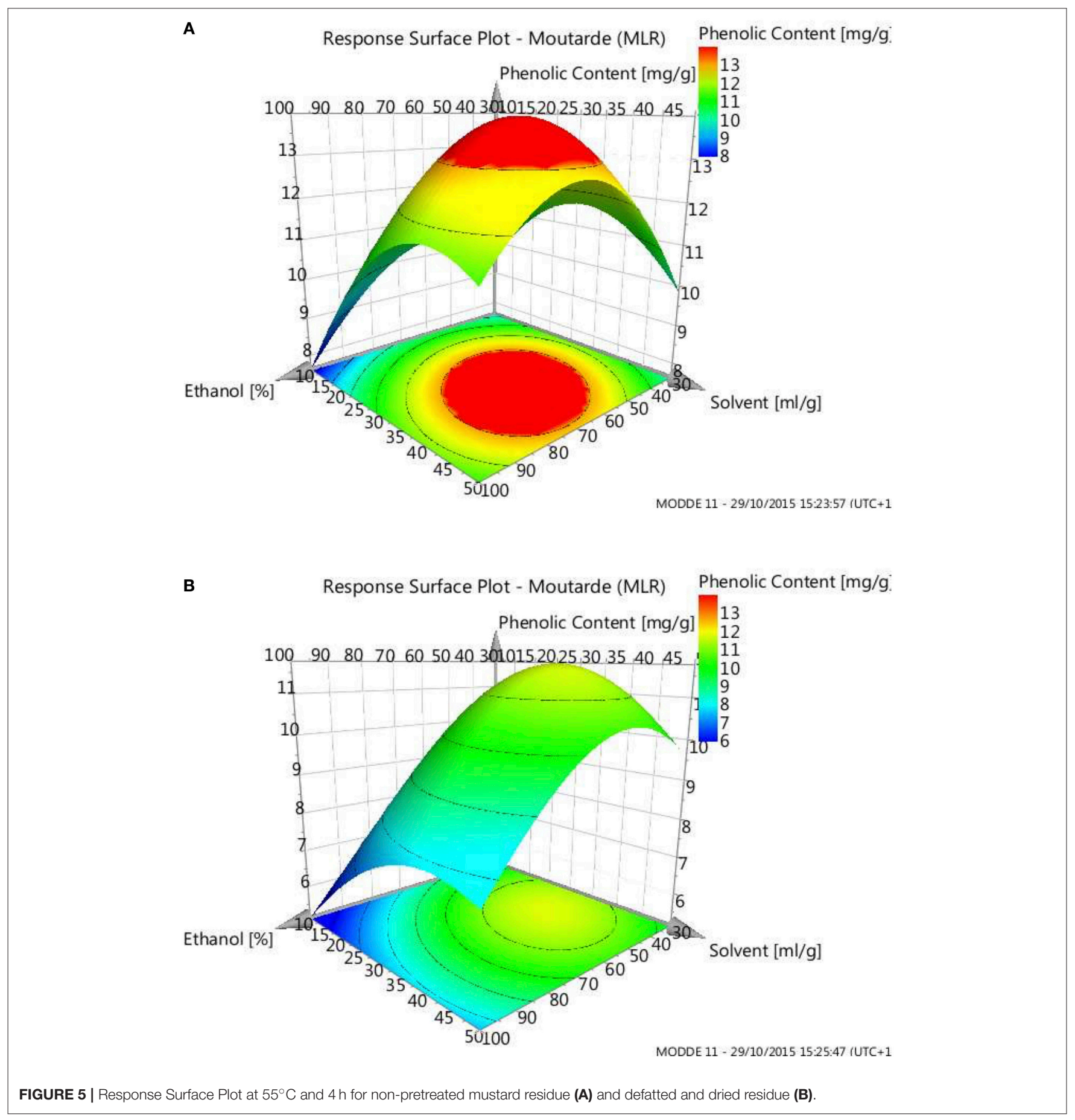


TABLE 4 | Validation of the model using optimal conditions for phenolics extraction.

\begin{tabular}{|c|c|c|c|c|c|c|c|c|}
\hline Entry & $\operatorname{Temp}\left({ }^{\circ} \mathrm{C}\right)$ & Time (h) & EtOH (\%) & $\mathrm{S} / \mathrm{M}(\mathrm{mL} / \mathrm{g})$ & $\mathbf{P t}$ & Pred. Phenolic Content (mg/g) & Model error $( \pm)$ & Exp. Phenolic Content (mg/g) \\
\hline 1 & 55 & 3.98 & 64.83 & 35.60 & $\mathrm{~N}$ & 13.80 & 1.65 & 11.40 \\
\hline 2 & 55 & 4 & 47 & 33.17 & DD & 11.83 & 1.11 & 10.56 \\
\hline 3 & 55 & 3.98 & 64.83 & 35.60 & $\mathrm{~N}$ & 13.80 & 1.65 & 13.71 \\
\hline 4 & 55 & 4 & 47 & 33.17 & DD & 11.83 & 1.11 & 10.98 \\
\hline
\end{tabular}

$$
\begin{aligned}
& \log (Y)=0.738685-0.0025656 \mathrm{X} 1+0.0815867 \mathrm{X} 2 \\
& \quad+0.00134211 \mathrm{X} 3+0.00199775 \mathrm{X} 4+0.0238607 \\
& \quad-0.0102209 \mathrm{X}^{2}-0.0000620895 \mathrm{X}^{2}+0.000184466 \mathrm{X} 4^{2} \\
& +0.0000641509 \mathrm{X} 1 \mathrm{X} 3+0.000156992 \mathrm{X} 1 \mathrm{X} 4 \\
& \quad+0.0000603143 \mathrm{X} 3 \mathrm{X} 4-0.000996512 \mathrm{X} 3
\end{aligned}
$$

As shown in Figure 4, the parameters with positive impact were mainly temperature (Temp) followed by time and without pretreatment $[\operatorname{Pre}(\mathrm{None})]$ and the interractions terms between temperature and solvent/matter ratio $(\mathrm{Temp} * \mathrm{~S} / \mathrm{M})$ and those of percentage of ethanol [Temp*\%EtOH, \%EtOH*S/M, $\% \mathrm{EtOH}^{*} \operatorname{Pre}($ None)]. On the contrary, the percentage of ethanol $(\% \mathrm{EtOH})$, the deffating and drying pretreatment [Pre(DD)], their interaction $\left[\% \mathrm{EtOH}^{*} \operatorname{Pre}(\mathrm{DD})\right]$ and the square terms (Time* Time, \%EtOH*EtOH and S/M*S/M) impacted negatively the phenolic content in the extract. Such findings can be easily explained by the fact that elevated temperatures, that increase molecular agitation, facilitate the extraction process, while time needs to be carefully monitored to allow complete extraction while avoiding secondary reactions or oxidation. Pretreating the biomass has a negative impact on the extraction, most likely due to the loss of part of the phenolic compounds (lipophilic) during the defatting process (Thiyam-Holländer et al., 2014). This lipophilic character for phenolics explains why a larger amount of ethanol is required to recover the lipophilic phenolics trapped in the fatty matter for untreated residues. It is noteworthy to mention that the use of pure ethanol as extracting solvent has been ruled out as it would not solubilize charged molecules such as sinapine.

Finally, external validations were performed in duplicates, using optima given by the model (Figure 5 and Table 4), the first without defatting (Table 4, entries 1 and 3) and the second after defatting and drying (Table 4, entries 2 and 4). Taking into consideration the model error, $\pm 1.65 \mathrm{mg} / \mathrm{g}$ DDM for none pretreated material and $\pm 1.11 \mathrm{mg} / \mathrm{g}$ DDM for dried and defatted matter, the optima were validated. One can notice that a better phenolic content can be achieved with crude matter, $13 \mathrm{mg} / \mathrm{g} \mathrm{DDM}$, than with defatted and dried one, $11 \mathrm{mg} / \mathrm{g}$ DDM, mainly by tuning the ethanol content of the extraction solution (65 and 47\%, respectively). Compared to the non-optimized extractions, the phenolic content extracted from crude residue was almost doubled (best result $7.28 \mathrm{mg} / \mathrm{g} \mathrm{DDM}$ ) whereas just a slight increase (7\%) was observed for the dried and defatted material. To summarize, this optimization allowed us to determine the best solvent mixture allowing the efficient extraction of the lipophilic phenolics trapped in the fatty matter present in the untreated residues. For the defatted and dried residues, as the fatty matter has been previously removed during the defatting process, the extent of the optimization remained limited as the lipophilic phenolics are more readily accessible and thus more easily extracted.

As each biomass is very specific (e.g., botanical species, growth conditions, treatment, type of extraction), comparing our results with the ones previously reported in literature is rather irrelevant. Dubie et al. (2013) who used a meal of Brassica juncea from an industrial source reported between 6 and $10 \mathrm{mg}$ $\mathrm{SAE} / \mathrm{g}$ depending on the extraction conditions (e.g., \%ethanol, temperature, ultrasound) and until $13.8 \mathrm{mg} / \mathrm{g}$ after 7 days in hot water. In another hand, Thiyam-Holländer et al. (2014) reported total phenolic contents between 11.5 and $42.3 \mathrm{mg} \mathrm{SAE} / \mathrm{g}$ after methanolic (70\%) extraction assisted by ultrasound on mustard flours. Assuming that flours are less processed than the mustard residues from industrial mustard production used in this study, one can consider that our optimized process is at least as efficient.

\section{CONCLUSION}

This study undoubtedly demonstrates that the way data from Folin-Ciocalteu analyses are reported and the choice of the standard are not trivial. Special attention should therefore be paid when performing and reporting such analyses.

By combining a preliminary extraction conditions screening and a Design of Experiment based on Response Surface Methodology, a very simple and eco-friendly process using only one extraction with ethanol and water without any pretreatment of an industrial co-product was optimized and allowed the obtention of a sinapine-rich phenolic extract. The latter can be used directly or after concentration as an antioxidant formulation, or further hydrolyzed to produce sinapic acid.

\section{AUTHOR CONTRIBUTIONS}

$\mathrm{AF}$ and FA conceived and managed the research. AF and GW performed the experiments. AT provided the technical guidelines for the RMS. AF, AT, and FA reviewed the results and provided the technical guidelines. AF wrote and drafted the article. AT and FA reviewed the article. FA approved the article. 


\section{ACKNOWLEDGMENTS}

We would like to thank the Region Grand Est, the Conseil Départemental de la Marne and the Grand Reims for their financial support, as well as Charbonneaux-Brabant for providing the mustard residue.

\section{REFERENCES}

Amarowicz, R., Wanasundara, U. N., Karamać, M., and Shahidi, F. (1996). Antioxidant activity of ethanolic extract of mustard seed. Food/Nahrung 40, 261-263. doi: 10.1002/food.19960400506

Baker, L. A., Horbury, M. D., Greenough, S. E., Allais, F., Walsh, P. S., Habershon, S., et al. (2016). Ultrafast photoprotecting sunscreens in natural plants. J. Phys. Chem. Lett. 7, 56-61. doi: 10.1021/acs.jpclett.5b02474

Dean, J. C., Ryoji, K., Walsh, P. S., Allais, F., and Zwier, T. S. (2014). Plant sunscreens in the UV-B: ultraviolet ppectroscopy of jet-cooled sinapoyl malate, sinapic acid, and sinapate ester derivatives. J. Am. Chem. Soc. 136, 14780-14795. doi: $10.1021 /$ ja5059026

Dubie, J., Stancik, A., Morra, M., and Nindo, C. (2013). Antioxidant extraction from mustard (Brassica juncea) seed meal using high-intensity ultrasound. J. Food Sci. 78, 542-548. doi: 10.1111/1750-3841.12085

Engels, C., Schieber, A., and Gänzle, M. G. (2012). Sinapic acid derivatives in defatted oriental mustard (Brassica juncea L.) seed meal extracts using UHPLCDAD-ESI-MSn and identification of compounds with antibacterial activity. Eur. Food Res. Technol. 234, 535-542. doi: 10.1007/s00217-012-1669-z

Enovation Chemicals (2018). Available online at: http://www.enovationchem. com/Products.asp?SEARCHTEXT $=530-59-6 \&$ searchbtn. $x=0 \&$ searchbtn. $y=0$ (Accessed July 23, 2018).

Huang, S., Huang, M., and Feng, B. (2012). Antioxidant activity of extracts produced from pickled and dried mustard (Brassica juncea coss. var. foliosa Bailey). Int. J. Food Prop. 15, 374-384. doi: 10.1080/10942912.2010.487628

Ishtiaque, S., Khan, N., Siddiqui, M., Siddiqi, R., and Naz, S. (2013). Antioxidant potential of the extracts, fractions and oils derived from oilseeds. Antioxidants 2, 246-256. doi: 10.3390/antiox2040246

Janvier, M., Ducrot, P.-H., and Allais, F. (2017a). Isocyanate-free synthesis and characterization of renewable poly(hydroxy)urethanes from syringaresinol. ACS Sustain. Chem. Eng. 5, 8648-8656. doi: 10.1021/acssuschemeng.7b01271

Janvier, M., Hollande, L., Jaufurally, A. S., Pernes, M., Ménard, R., Grimaldi, M., et al. (2017b). Syringaresinol: a renewable and safer alternative to bisphenol A for epoxy-amine resins. ChemSusChem 10, 738-746. doi: $10.1002 /$ cssc. 201601595

Jaufurally, A. S., Teixeira, A. R. S., Hollande, L., Allais, F., and Ducrot, P.-H. (2016). Optimization of the laccase-catalyzed synthesis of $( \pm)$-syringaresinol and study of its thermal and antiradical activities. Chem. Select 1, 5165-5171. doi: 10.1002/slct.201600543

Kozłowska, M., Gruczyńska, E., šcibisz, I., and Rudzińska, M. (2016). Fatty acids and sterols composition, and antioxidant activity of oils extracted from plant seeds. Food Chem. 213, 450-456. doi: 10.1016/j.foodchem.2016.06.102

Li, C., Tang, Z., Huang, M., Tao, N., Feng, B., and Huang, S. (2012). Antioxidant efficacy of extracts produced from pickled and dried mustard in rapeseed and peanut oils. J. Food Sci. 77, 394-400. doi: 10.1111/j.1750-3841.2011.02606.x

Luo, J., Liu, Y., Yang, S., Flourat, A. L., Allais, F., and Han, K. (2017). Ultrafast barrierless photoisomerization and strong ultraviolet absorption of photoproducts in plant sunscreens. J. Phys. Chem. Lett. 8, 1025-1030. doi: 10.1021/acs.jpclett.7b00083

Mayengbam, S., Aachary, A., and Thiyam-Holländer, U. (2014). Endogenous phenolics in hulls and cotyledons of mustard and canola: a comparative study on its sinapates and antioxidant capacity. Antioxidants 3, 544-558. doi: 10.3390/antiox3030544

McCue, P., Kwon, Y. I., and Shetty, K. (2005). Anti-amylase, anti-glucosidase and anti-angiotensin I-converting enzyme potential of selected foods. J. Food Biochem. 29, 278-294. doi: 10.1111/j.1745-4514.2005.00020.x

\section{SUPPLEMENTARY MATERIAL}

The Supplementary Material for this article can be found online at: https://www.frontiersin.org/articles/10.3389/fsufs. 2019.00012/full\#supplementary-material

OCDE/FAO (2015). OCDE/Organisation des Nations Unies pour l'Alimentation et l'Agriculture (2015), Perspectives Agricoles de l'OCDE et de la FAO 2015. Paris: OCDE. doi: 10.1787/agr_outlook-2015-fr

Park, S. Y., Jang, H. L., Lee, J. H., Choi, Y., Kim, H., Hwang, J., et al. (2017). Changes in the phenolic compounds and antioxidant activities of mustard leaf (Brassica juncea) kimchi extracts during different fermentation periods. Food Sci. Biotechnol. 26, 05-12. doi: 10.1007/s10068-017-0014-5

Santos, J., Oliveira, M. B. P. P., Ibáñez, E., and Herrero, M. (2014). Phenolic profile evolution of different ready-to-eat baby-leaf vegetables during storage. J. Chromatography A 1327, 118-131. doi: 10.1016/j.chroma.2013.12.085

Smyk, B. (2015). Spectral properties of sinapine in water environment. J. Mol. Liquids 208, 1-4. doi: 10.1016/j.molliq.2015.03.058

Song, F. L., Gan, R. Y., Zhang, Y., Xiao, Q., Kuang, L., and Li, H. B. (2010). Total phenolic contents and antioxidant capacities of selected chinese medicinal plants. Int. J. Mol. Sci. 11, 2362-2372. doi: 10.3390/ijms11062362

Surendra, S., Godara, S. L., Gangopadhayay, S., and Jadon, K. S. (2012). Induced resistance against alternaria brassicae blight of mustard through plant extracts. Arch. Phytopatho. Plant Protec. 45, 1705-1714. doi: 10.1080/03235408.2012.702465

Szydlowska-Czerniak, A., Tulodziecka, A., Karlovits, G., and Szlyk, E. (2015). Optimisation of ultrasound-assisted extraction of natural antioxidants from mustard seed cultivars. J. Sci. Food Agr. 95, 1445-1453. doi: 10.1002/jsfa.6840

Teixeira, A. R. S., Flourat, A. L., Peru, A. M. M., Brunissen, F., and Allais, F. (2016). Lipase-catalyzed baeyer-villiger oxidation of cellulose-derived levoglucosenone into (S)- $\gamma$-hydroxymethyl- $\alpha, \beta$-butenolide: optimization by response surface methodology. Front. Chem. 4, 1-11. doi: 10.3389/fchem.2016.00016

Terpinc, P., Čeh, B., Ulrih, N. P., and Abramovič, H. (2012). Studies of the correlation between antioxidant properties and the total phenolic content of different oil cake extracts. Ind. Crops Prod. 39, 210-217. doi: 10.1016/j.indcrop.2012.02.023

Thangi, J., Shashitha, K. N., Ashiwini, H. A., and Shlini, P. (2016). A correlation study of antioxidant potential's from synapis alba. Indo Am. J. Pharm. Sci. 3, 234-239. Available online at: http://www.iajps.com/pdf/march2016/7. Synapis\%20alba.pdf

Thiyam, U., Kuhlmann, A., Stöckmann, H., and Schwarz, K. (2004). Prospects of rapeseed oil by-products with respect to antioxidative potential. Comptes Rendus Chim. 7, 611-616. doi: 10.1016/j.crci.2004.02.011

Thiyam-Holländer, U., Aladedunye, F., Logan, A., Yang, H., and Diehl, B. W. K. (2014). Identification and quantification of canolol and related sinapate precursors in indian mustard oils and canadian mustard products. Eur. J. Lipid Sci. Technol. 116, 1664-1674. doi: 10.1002/ejlt.201400222

Yang, M., Zheng, C., Zhou, Q., Liu, C., Li, W., and Huang, F. (2014). Influence of microwaves treatment of rapeseed on phenolic compounds and canolol content. J. Agri. Food Chem. 62, 1956-1963. doi: 10.1021/jf4054287

Conflict of Interest Statement: The authors declare that the research was conducted in the absence of any commercial or financial relationships that could be construed as a potential conflict of interest.

Copyright (c) 2019 Flourat, Willig, Teixeira and Allais. This is an open-access article distributed under the terms of the Creative Commons Attribution License (CC BY). The use, distribution or reproduction in other forums is permitted, provided the original author(s) and the copyright owner(s) are credited and that the original publication in this journal is cited, in accordance with accepted academic practice. No use, distribution or reproduction is permitted which does not comply with these terms. 